\title{
„Dysfunktionale Demokratie(n): Merkmale, Ursachen und Folgen“. Digitale Jahrestagung des AK Demokratieforschung der DVPW am 11. März 2021
}

\section{Toralf Stark - Susanne Pickel • Norma Osterberg-Kaufmann • Christoph Mohamad-Klotzbach}

Die digitale Jahrestagung 2021 des Arbeitskreises Demokratieforschung widmete sich dem Konzept der dysfunktionalen Demokratie. Die leitende Fragestellung lautete: Was sind Merkmale dysfunktionaler Demokratien, worin sind mögliche Ursachen zu finden und was sind die Folgen? Ziel der Jahrestagung war es zu eruieren, wann, wo und warum es in Demokratien zu weitreichenden Funktionsstörungen kommt.

Hintergrund der Tagung war die Feststellung, dass der Begriff der dysfunktionalen Demokratie in der wissenschaftlichen Literatur durchaus auftaucht, gleichzeitig jedoch weitgehend undefiniert ist. Oftmals wird er mit dem Begriff der defekten Demokratie (Merkel) oder der defizitären Demokratie (Lauth) gleichgesetzt. Auf Basis strukturfunktionalistischer und systemtheoretischer Überlegungen (u.a. Almond, Easton, Parsons) könnte man das Konzept der dysfunktionalen Demokratie

Dr. T. Stark · Prof. Dr. S. Pickel

Institute of Political Science, University of Duisburg-Essen, Lotharstr. 63, 47057 Duisburg,

Deutschland

Dr. T. Stark

E-Mail: toralf.stark@uni-due.de

Prof. Dr. S. Pickel

E-Mail: susanne.pickel@uni-due.de

Dr. N. Osterberg-Kaufmann

Department of Social Sciences, Humboldt-Universität zu Berlin, Unter den Linden 6, 10099 Berlin, Deutschland

E-Mail: norma.osterberg-kaufmann@hu-berlin.de

Dr. C. Mohamad-Klotzbach $(\varangle)$

Institute of Political Science and Sociology, University of Würzburg,

Wittelsbacherplatz 1, 97074 Würzburg, Deutschland

E-Mail: ch.mohamad@uni-wuerzburg.de 
jedoch nicht nur in Bezug auf die Struktur definieren (polity), sondern auch auf politische Prozesse (politics), politische Entscheidungen (policy outputs) sowie ihre Wirkungen (policy outcomes) erweitern. Zudem rücken Veränderungen innerhalb der politischen Kultur sowie anderer gesellschaftlicher Subsysteme in den Blick, die somit zu Dysfunktionen der politischen Institutionen sowie der Prozesse führen können. Die Beiträge der Tagung beleuchteten hierbei unterschiedliche Facetten des Phänomens.

Mit ihrem Beitrag „Dysfunktionale Demokratie(n): Ein konzeptioneller Vorschlag“ eröffneten Christoph Mohamad-Klotzbach (Universität Würzburg) und Norma Osterberg-Kaufmann (HU Berlin) die Jahrestagung. Darin fassten sie den im öffentlichen und akademischen Diskurs eher diffus verwendeten Begriff der dysfunktionalen Demokratie konzeptionell und entwickelten ein heuristisches Instrument zur Strukturierung der empirischen Forschung, das als breiterer theoretischer Rahmen für empirische Arbeiten dienen kann. Grundlage der Überlegungen sind strukturfunktionalistische und systemtheoretische Ansätze. In der Logik des Systemerhalts sind Demokratien der Argumentation von Mohamad-Klotzbach und Osterberg-Kaufmann zufolge solange funktional, wie es ihnen gelingt sich an die permanent ändernden Umweltanforderungen adäquat anzupassen. Dysfunktional werden Demokratien dann, wenn die Anpassungsmechanismen nicht mehr funktionieren und sich spezifische Dysfunktionen verfestigen. Ebenso ist es vorstellbar, dass bestimmte Anpassungen ein demokratisches System nicht stabilisieren, sondern zu einer De-Demokratisierung bzw. Autokratisierung des Systems beitragen.

Der zweite Vortrag von Svend-Erik Skaaning (Aarhus University) zum Thema „More State Repression in Hybrid Regimes? A Global Assessment, 1800-2016“, ging der Frage nach, ob es einen Zusammenhang zwischen unterschiedlichen Regimetypen und dem Ausmaß an staatlicher Repression gibt. Dabei prüfte Skaaning die These, ob gemäß dem Forschungstand staatliche Repression in hybriden Regimen tatsächlich häufiger vorkommt als in autoritären und demokratischen Regimen. In seiner umfassenden empirischen Analyse berücksichtige er auch historische Regimevarianten. Auf Basis einer kategorialen Unterscheidung politischer Regime, insbesondere der Ausdifferenzierung autoritärer und hybrider Regime, konnte er diesen Zusammenhang nicht belegen. Vielmehr stellen sich autoritäre Regime als wesentlich repressiver dar als die hybriden Regimeformen.

Das zweite Panel begann mit dem Vortrag von Oliver Schlenkrich und Lukas Lemm (beide Universität Würzburg), die sich mit der Frage nach der Entstehung von spezifischen Dysfunktionen in den politischen Strukturen von Demokratien auseinandersetzten. Zunächst unterschieden sie auf Basis der Demokratiematrix vier Subtypen von Demokratie (balanced, illiberal, inegalitarian und unaccountable democracy). Während die erste Variante in den Dimensionen Freiheit, Gleichheit und Kontrolle voll ausgeprägt ist, zeigen die übrigen ein Defizit in jeweils einer Dimension und bilden so drei verschiedene Varianten defizitärer Demokratie ab. In einem zweiten Schritt versuchten sie auf Basis verschiedener Theoriestränge (Modernization Theory, Power Dispersion Theory, Partisan Theory, Institutionalism, Statehood, External Influences) Varianten im Hinblick auf die institutionellen Muster zu erklären. Ziel ist es herauszufinden, ob es spezifische Faktoren gibt, die diese Varianz jeweils erklären können. Im Ergebnis zeigten sie, dass häufig genutzte 
Erklärungsfaktoren aus der Transformationstheorie - Staatlichkeit, Parteieffekte, Regierungstyp, ethnische und sprachliche Fragmentierung, Globalisierung und soziale Ungleichheit - unterschiedliche Effekte auf die Dimensionen der Demokratie haben und deshalb mit bestimmten Subtypen von Demokratie korrespondieren.

Im zweiten Vortrag dieses Panels widmeten sich Wolfgang Muno und Christian Pfeiffer (beide Universität Rostock) den Dysfunktionen der chilenischen Demokratie und untersuchten eingehender die Rolle der politischen Eliten. Ausgehend von der Erkenntnis, dass es sich bei der politischen Elite Chiles um eine recht homogene Gruppe handelt, die in einer eigenen ,Wirklichkeit“ lebt, nahmen sie an, dass auch deren Demokratieverständnis als einheitlich betrachtet werden kann. Auf Basis von mehreren hundert Meinungsartikeln aus zwei chilenischen Zeitungen, den Hauptinformationsquellen der Chilen:innen über die Ansichten ihrer politischen Elite analysierten die beiden auf Grundlage des Demokratiekonzepts der Demokratiematrix die vorliegenden Demokratieverständnisse. Wie vermutet, liegt innerhalb der chilenischen politischen Elite ein recht einheitliches Demokratieverständnis vor, dass sich v. a. darauf fokussiert, dass die Demokratie im Schumpeterschen Sinne als eine repräsentative Demokratie zu verstehen ist, bei der die Bevölkerung lediglich alle vier Jahre zur Wahl geht, darüber hinaus jedoch unpolitisch bleibt. Die andauernden Proteste in Chile sind allerdings ein Anzeichen dafür, dass es hier offenbar eine Diskrepanz in den Demokratieverständnissen zwischen der Elite und der Bevölkerung gibt.

Im dritten Panel stand die Analyse von populistischen Parteien und Einstellungen im Vordergrund. Carsten Wegscheider (Universität Salzburg), Toralf Stark und Susanne Pickel (beide Universität Duisburg-Essen) untersuchten, ob spezifische Demokratieverständnisse die Wahl populistischer Parteien in Europa erklären können. Sie betrachteten hierfür auf Basis der European Value Study (EVS) 19 Länder, in denen links- wie rechtspopulistische Parteien eine Rolle spielen. Als zentrale erklärende Variablen fokussierten sie neben dem liberalen, autoritären und substantiellen Demokratieverständnis auch auf demokratisches Wissen, dass sich durch eine Zustimmung zu den Merkmalen der liberalen Demokratie bei gleichzeitiger Ablehnung autoritärer Merkmale definiert. Die Ergebnisse zeigten, dass den Erwartungen entsprechend, Personen mit einer Präferenz für Umverteilung und umfassende staatliche Unterstützung eher zu (populistischen) linksradikalen Parteien neigen, wohingegen ein autoritäres Demokratieverständnis eher die Präferenz für (populistische) rechtsradikale Parteien hervorruft. Ergänzend stellten sie fest, dass die Unterschiede in der Präferenz auf die Hostideologie und nicht auf populistische Einstellungen im Allgemeinen zurückzuführen sind, da die Unzufriedenheit mit der Demokratie sowie ein geringeres Vertrauen in politische Institutionen bei beiden Unterstützergruppen vorzufinden ist.

Im zweiten Vortrag des dritten Panels zum Thema „Populistische Bürger:innen Fundamentale Gegner der liberalen Demokratie oder enttäuschte Unterstützer“ präsentierte Alexander Wuttke (Universität Mannheim) eine Studie, die er gemeinsam mit Christian H. Schimpf (University of Alberta) und Harald Schoen (Universität Mannheim) verfasst hat. Die Autoren vergleichen darin Einstellungen von Bürger:innen in vier Ländern (Großbritannien, Deutschland, Frankreich und Schweiz) zu unterschiedlichen Aspekten von Demokratie und prüfen, inwiefern sich Bürger:innen 
mit populistischen Einstellungen von Bürger:innen mit nicht-populistischen Einstellungen unterscheiden. Dabei fanden Wuttke, Schimpf und Schoen im Rahmen ihres Fallvergleichs heraus, dass es durchaus Unterschiede in den Ländern gibt. Während in Deutschland beispielsweise bei den meisten Demokratieeinstellungen deutliche Unterschiede zwischen den Populisten und Nicht-Populisten erkennbar sind, ist für Großbritannien durchgehend eine größere Übereinstimmung zwischen den Gruppen feststellbar.

Das letzte Panel eröffnete Susanne Pickel (Universität Duisburg-Essen) mit einem Vortrag zum Thema „Malfunctions of Democratic Principles. Misunderstanding Freedom of Expression“. Auf Basis eines neuen Messinstruments aus einer repräsentativen Bevölkerungsumfrage untersucht sie die Diskrepanz zwischen einem liberalen und einem populistischen Verständnis von Meinungsfreiheit. Die Befunde zeigen, dass die Fehlwahrnehmung dessen, was Meinungsfreiheit bedeutet, durch eine autoritäre Grundhaltung, geringe Toleranz gegenüber der Kultur von Fremdgruppen, Nativismus, Offenheit für Populismus, mangelnde soziale Anerkennung und Vertrauen in soziale Medien (als Proxy für deren Nutzung) erklärt werden kann. Die abschließende Untersuchung des Zusammenhangs zwischen Meinungsfreiheit und der Wahlpräferenz - insbesondere im Hinblick auf die AfD zeigt, dass nur in Westdeutschland ein signifikanter Zusammenhang zwischen der Wahl bzw. der Identifikation mit der AfD und der Fehlinterpretation des Rechtes auf freie Meinungsäußerung festgestellt werden kann. Die Wähler:innen und Sympathisant:innen der LINKEN wussten hingegen in beiden Landesteilen überdurchschnittlich häufig, was Meinungsfreiheit wirklich bedeutet. Im Ergebnis konnte gezeigt werden, dass falsche Annahmen über den Kern der Meinungsfreiheit häufig nicht zufällig geäußert werden, sondern mit antidemokratischen Grundhaltungen zusammenfallen und auf Meinungsführerschaft ohne Widerspruch abzielen.

Im letzten Vortrag der Tagung widmete sich Lasse Egendal Leipziger (Aarhus University) der Frage: ,To what extent and how does democracy reduce ethnic socioeconomic inequalities?" Er stellte fest, dass es relativ wenig Forschung zum Thema ethnischer Ungleichheiten in Demokratien gibt und seine Studie hier einen Beitrag leisten kann. Die theoretische Grundlage fußt auf drei beobachteten Mechanismen bei der Etablierung demokratischer politischer Regime: Die Regierungen werden responsiver gegenüber zuvor vernachlässigten Gruppen, bürgerliche Freiheiten und die freie Arbeit von NGOs und Medien verschaffen den deprivierten Gruppen mehr Einfluss und demokratische Verfahren schaffen mehr Chancengleichheit zwischen den Bevölkerungsgruppen. Egendal Leipziger verfolgte die Annahmen, dass demokratische Systeme die Ungleichheit zwischen ethnischen Gruppen reduzieren und dies insbesondere dort gilt, wo diese Ungleichheit unter autokratischer Herrschaft zuvor besonders hoch ausgefallen ist. Im Ergebnis konnte er belegen, dass die Ungleichheit auf ethnischer Basis durch die Demokratisierung deutlich reduziert und gemildert werden kann.

Die Abschlussdiskussion widmete sich nochmals der konzeptionellen Dimension von dysfunktionalen Demokratien von Mohamad-Klotzbach und Osterberg-Kaufmann. Dabei wurde diskutiert, ob und wie das vorgestellte Konzept stärker auf den politischen Prozess und die politischen Entscheidungen fokussiert werden könnte oder wie eine Differenzierung zwischen Makro-, Meso- und Mikroebene die kon- 
zeptionelle Klarheit des Begriffs stärkt. Zudem wurden grundsätzliche Restriktionen des strukturfunktionalistischen Ansatzes kritisiert: Insbesondere den Akteuren müsste mehr Bedeutung beigemessen werden, als es die strukturfunktionalistische Logik nahelegt.

Konsens bestand jedoch in zweierlei Hinsicht: Erstens, dass es sinnvoll ist, den Begriff der dysfunktionalen Demokratie konzeptionell genauer zu erfassen und ihn von bereits existierenden Begriffen abzugrenzen. Zweitens, dass die im Rahmen der Tagung vorgestellten empirischen Befunde wichtige Erkenntnisse für die politischen Entwicklungen in den demokratischen Regimen liefern und diverse Dysfunktionen in Demokratien in den Blick rückten. Schließlich war man sich einig, dass es weiterer Forschung bedarf, um die noch offenen sowie neu aufgeworfenen Fragen zu klären.

Funding Open Access funding enabled and organized by Projekt DEAL.

Open Access Dieser Artikel wird unter der Creative Commons Namensnennung 4.0 International Lizenz veröffentlicht, welche die Nutzung, Vervielfältigung, Bearbeitung, Verbreitung und Wiedergabe in jeglichem Medium und Format erlaubt, sofern Sie den/die ursprünglichen Autor(en) und die Quelle ordnungsgemäß nennen, einen Link zur Creative Commons Lizenz beifügen und angeben, ob Änderungen vorgenommen wurden.

Die in diesem Artikel enthaltenen Bilder und sonstiges Drittmaterial unterliegen ebenfalls der genannten Creative Commons Lizenz, sofern sich aus der Abbildungslegende nichts anderes ergibt. Sofern das betreffende Material nicht unter der genannten Creative Commons Lizenz steht und die betreffende Handlung nicht nach gesetzlichen Vorschriften erlaubt ist, ist für die oben aufgeführten Weiterverwendungen des Materials die Einwilligung des jeweiligen Rechteinhabers einzuholen.

Weitere Details zur Lizenz entnehmen Sie bitte der Lizenzinformation auf http://creativecommons.org/ licenses/by/4.0/deed.de. 